\title{
Akademische Psychotherapie, Psychotherapiewissenschaft und Experimentalhermeneutische Laborforschung
}

\author{
Eine Ergänzung zu Markus Erismanns \\ "Der Wissenschaftsbegriff der Psychotherapiewissenschaft»
}

\author{
Kurt Greiner \\ Psychotherapie-Wissenschaft 9 (2) 20-28 2019 \\ www.psychotherapie-wissenschaft.info \\ CC BY-NC-ND \\ https://doi.org/10.30820/1664-9583-2019-2-20
}

\begin{abstract}
Zusammenfassung: Dieser Kommentar zu Markus Erismanns Essay mit dem Titel «Der Wissenschaftsbegriff der Psychotherapiewissenschaft» ist als Argumentationsergänzung konzipiert, in der es zu zeigen gilt, was eine wissenschaftshermeneutisch konzeptualisierte Psychotherapiewissenschaft für die akademische Statuskarriere der Psychotherapie potenziell zu leisten vermag. So wird im Anschluss an theoretische Erörterungen sowie methodologische Darlegungen anhand einer exemplarischen Modellanalyse demonstriert, wie jene innovative Grundlagenforschungspraxis funktioniert, die dazu beitragen kann, dass Psychotherapie zu einer genuinen Einzelwissenschaft avanciert.
\end{abstract}

Schlüsselwörter: Akademische Psychotherapie, Markus Erismann, Experimental- und Imaginativhermeneutische Psychotherapiewissenschaft, Greiner-Jandl-Initiative, Therapieschulenforschung, Therapieschulendialog (TSD), Experimentelle Trans-Kontextualisation (ExTK), Sigmund Freud PrivatUniversität Wien (SFU)

\section{Vorbemerkung}

Einer freundlichen Einladung nachkommend, Markus Erismanns Essay mit dem Titel «Der Wissenschaftsbegriff der Psychotherapiewissenschaft» zu kommentieren, sei informativ vorausgeschickt, dass ich seit zwölf Jahren an der Sigmund Freud PrivatUniversität Wien (SFU) im Wissenschaftsfach Psychotherapiewissenschaft (PTW) lehre und forsche und dort gemeinsam mit Martin Jandl das 1. Experimentalhermeneutische Labor der PTW gründete. An der im Jahre 2005 in Lehrbetrieb gegangenen SFU Wien kann man Psychotherapie nicht nur praktisch erlernen, sondern darüber hinaus auf universitärem Niveau studieren. Die SFU Wien gilt weltweit als jene Universität, an der die Psychotherapie unter dem Terminus PTW erstmals als eigenständiges Studienfach in Erscheinung trat und damit die lang ersehnte Aufnahme in den Kreis der etablierten Einzelwissenschaften im akademisch-institutionalisierten Forschungsbetrieb fand.

\section{Akademische Wissenschaftlichkeit und Psychotherapie}

Die ambitionierten Bemühungen des Züricher Philosophen Markus Erismann um die Frage nach dem «speziellen, schulenübergreifenden Wissenschaftsbegriff der Psychotherapiewissenschaft» münden, nach einigen ar- gumentativen Zwischenstationen, in der Auseinandersetzung mit dem Terminus «Reflexion», der sich in dieser Begründungsdiskussion als methodische Kern-Kategorie entpuppt. Der Reflexionsbegriff taucht in seinem Text an mehreren Stellen auf, unter anderem auch kombinativ als «kritische Reflexion», «Selbstreflexion» oder «Reflexionsprozess", und im Fazit des Abrundungskapitels wird schliesslich dessen zentraler Stellenwert klar und deutlich hervorgehoben, wenn es dort in den beiden letzten Sätzen heisst: «In der Psychotherapiewissenschaft ist der gemeinsame, dialogische Reflexionsprozess von PatientIn und TherapeutIn Ort des therapeutischen Heilens und der Wissensgenerierung. Die Methode der Reflexion macht die Eigenständigkeit und Wissenschaftlichkeit der Psychotherapiewissenschaft aus» (Erismann, 2019, S. 16 i.d. Heft).

Erismanns Argumentation ist insofern zuzustimmen, als dass das Funktionsprinzip Reflexion gewiss konstitutiv für sämtliche psychotherapeutische Praxen des Heilens und Wissensgenerierens ist. In den einzelnen psychotherapeutischen Disziplinen - damit sind hier die Schulen bzw. Modalitäten der Psychotherapie gemeint - haben sich im Verlauf der modalitätsgeschichtlichen Entwicklungen schulentypische Instrumentarien des systematischen Reflektierens und Sinndeutens, des Interpretierens und Verstehens herausgebildet und ausdifferenziert, die freilich nicht auf eine gemeinsame Strukturlogik oder auf ein für alle verbindliches paradigmatisches Grundprinzip 
rekurrieren (Merkmal der Inkommensurabilität). Ganz in diesem Sinne muss die multikonzeptuelle und methodenpluralistische Wissenschaftskultur Psychotherapie im Rahmen ihrer differenten Disziplinen (Psychoanalyse, Analytische Psychologie, Gestalttherapie, Systemische Psychotherapie, Verhaltenstherapie etc.) auf je spezifische Weisen adäquat funktionieren, sodass die methodische Schaffung von Wissen garantiert ist.

Nun gilt es aber zu berücksichtigen, dass es dem abendländischen Kulturphänomen Wissenschaft seit Anbeginn, nebst der methodischen Schaffung von Wissen, immer auch noch um die methodische Gewinnung von Erkenntnis ging (Wallner, 1992a; 2002). Mithin ist Akademische Wissenschaft, im Unterschied zu anderen, mehr oder weniger methodenbasierten Wissensformen (wie etwa esoterisches Wissen, religiöses Wissen, spiritistisches Wissen, lebensweltliches Wissen, fremdkulturelles Wissen etc.), durch den Anspruch auf Wissen in technisch-funktionaler Hinsicht (1. Konstitutionskriterium) und auf Erkenntnis in kritisch-reflexiver Hinsicht (2. Konstitutionskriterium) charakterisiert, was für die Psychotherapie Folgendes bedeutet: Beansprucht Psychotherapie nicht mehr, als dass sie adäquat funktioniert, degradiert sie sich selbst auf eine wissenschaftliche Heilungs- oder Gesundungstechnik und schafft damit bloss methodisches Wissen über instrumentell nützliche Umgangsweisen mit ihren Gegenständen in therapeutischer Intention. Möchte sich Psychotherapie aber als akademische Einzelwissenschaft verstehen, muss sie sich darüber hinaus auch um die systematische Produktion vertiefter Einsichten in die komplexen Strukturen ibrer professionellen Aktivitäten bemühen, womit sie schliesslich erst differenzierte Erkenntnisse hinsichtlich impliziter Voraussetzungen und Bedingungen ihrer methodischen Wissenschaftsakte gewinnt. Das heisst, die schulen- bzw. modalitätsbasierten psychotherapeutischen Disziplinen müssen ihre je spezifischen Funktionsweisen mit wissenschaftshermeneutischen Strategien kritisch untersuchen, damit sich sukzessive ein zunehmend differenzierter werdendes Niveau des Selbstverstehens hinsichtlich psychotherapeutischer Denk- und Handlungsmodi herauskristallisieren kann, wodurch nicht zuletzt deren Sinngrenzen und Verbindlichkeitsdimensionen sichtbar, kommunizierbar, kritisierbar und diskutierbar werden.

Interessanterweise besitzt die Psychotherapie aufgrund ihrer «bewusst multiparadigmatischen» (Wagner, 1996, S. 245) Forschungs- und Praxisstruktur einen prinzipiellen reflexionsmethodologischen Startvorteil und hat insofern in technikreflexiver Hinsicht sogar eine richtungsweisende Vorbildfunktion für andere Wissenschaftsfächer. Wissenschaftshermeneutische Reflexionen des professionellen Denkens und Handelns können im Falle der Psychotherapie im eigenen Wissenschaftsrevier durchgeführt werden, weil dieses polykonzeptuell, methodenpluralistisch und theorienheterogen strukturiert ist und daher ideale Möglichkeiten für erkenntnisevozierende Bemühungen im oben angesprochenen Sinne potenziell bereitstellt, die in anderen Fachgebieten nicht automatisch gegeben sind. Dieser Sachverhalt einer innerwissenschaftlichen Vielschichtigkeitsund Fremdartigkeitsstruktur, der in jenem Ausmass, das für die Psychotherapie kennzeichnend ist, allen etablierten Wissenschaftsfächern unmöglich zu sein scheint, stellt für die Psychotherapie eben nicht nur ein fundamentales methodologisches Abgrenzungskriterium gegenüber anderen Forschungsrichtungen dar, sondern lässt die charakteristische Struktur der Psychotherapie als mustergültige Bedingung für wissenschaftsreflexive Erkenntnisunternehmungen via wechselseitige Bezugnahmen zwischen den einzelnen Disziplinen begreifen (Therapieschulen-Interdisziplinarität). Faktisch liegt in keinem anderen wissenschaftlichen Fach eine dermassen hohe fachimmanente Reflexions-Provokation strukturbedingt vor, wie in der Psychotherapie. Allein dieses Qualitätsmerkmal begünstigt die kritisch-reflexive Erkenntnisproduktion, die das 2. Konstitutionskriterium für Akademische Wissenschaftlichkeit, nebst der technischfunktionalen Wissensproduktion (1. Konstitutionskriterium), repräsentiert.

\section{Zum Ansatz einer Psychotherapiewissenschaft, die für die Akademisierungsambition der Psychotherapie von Nutzen ist}

Was also die Problematik der Wissenschaftlichkeit der Psychotherapie im akademischen Sinne betrifft, bedürfen die an sich stringenten Überlegungen Erismanns einer Ergänzung. Dabei muss zunächst eine Begriffsdifferenzierung zwischen den Termini Psychotherapie (PT), Psychotherapiewissenschaft (PTW) und Akademische Psychotherapie (APT) dergestalt vorgenommen werden, dass sich die Frage sinnvoll stellen lässt, ob eine Art von PTW denkbar ist, die dazu beitragen kann, dass PT zur APT avanciert.

Auf Initiative meines Kollegen Martin Jandls und mir ist im Jahr 2008 im institutionalisierten Rahmen der SFU Wien mit der Gründung des 1. Experimentalhermeneutischen Labors der PTW ein Grundlagenforschungsprogramm in Schwung gekommen, das für eine positive Antwort auf genau diese Frage steht (Greiner et al., 2009, 2010; Greiner \& Jandl, 2012, 2015; Greiner, 2011, 2012, 2013a, 2015a, b). Zumindest belegen die vielen Aktivitäten am Institut für Hermeneutische Therapieschulenforschung und Therapieschulendialog (Fakultät PTW), dass die geforderte wissenschaftshermeneutische Reflexion der spezifischen Funktionsweise einer psychotherapeutischen Disziplin sowohl über dialogische Begegnungen mit anderen Psychotherapieschulen als auch über systematische Konfrontationen mit künstlerischen Medien oder sonstigen kulturellen Sinngebilden (Objektivationen) nachweislich gelingt. Für die methodische Umsetzung und Durchführung solcher Bezugnahmen wurden im Forschungsprogramm der Experimental- und Imaginativhermeneutischen PTW theorienanalytische Werkzeuge entwickelt, bei denen es sich um innovative, teilweise sogar radikal-kreative (bizarrosophische) Techniken des Sinnverstehens handelt, die allesamt auf dem kulturkonstruktivistischen Grundgedanken des irritationslogischen «Verfremdens» nach Wallner basieren (Greiner, 2007, 2013a; Greiner \& Jandl, 2012, 2015; Greiner et al., 2013; Jandl, 2006; Wallner, 1992a, b, 2002). 
Diese theorienanalytischen Reflexionsmethoden können dabei den folgenden zwei grossen Verfahrensgruppen zugeordnet werden: a) Experimental-und Imaginativhermeneutische Verfahren für den Dialog zwischen psychotherapeutischen Disziplinen sowie b) Experimental-und Imaginativhermeneutische Verfahren für den Dialog mit künstlerischen Medien. Als Instrumentarien der Verfahrensgruppe a) gelten die Experimentelle Trans-Kontextualisation im Standardisierten Therapieschulendialog (ExTK/TSD), das Intertherapeutische Text-Puzzle (ITTP), der Intertherapeutische Bild-Prozess (ITBP) sowie die Intertherapeutischen Medien-Spiele (ITMS) in ihren drei inter-therapeutischen Verfahrensmodi Psycho-MimikAnalyse (PMiA), Psycho-Musik-Analyse (PMuA) und Psycho-Tanz-Analyse (PTA) (Greiner, 2012, 2013a,d; Greiner \& Jandl, 2012).

Als Analysewerkzeuge der Verfahrensgruppe b) sind das Psycho-Text-Puzzle (P-T-P), der Psycho-Bild-Prozess $(P B P)$, das kleine und das grosse Psycho-Bild-Spiel $(P B S / k, P B S / g$ ) sowie die (analog zu den ITMS ebenso in drei Verfahrensvarianten konzeptualisierten) PsychoMedien-Spiele (PMS) zu nennen (Greiner \& Jandl, 2012, 2015; Greiner et al., 2013; Greiner, 2013b, c, 2014).

Es sei ausdrücklich betont, dass die Greiner-JandlInitiative im Zeichen des programmatischen Imperativs «Therapieschulenforschung von innen!» bzw. «Therapieschulenforschung von und für Psychotherapeut/innen!» angesetzt ist. Damit soll klar gesagt werden, dass mit diesen neuartigen, aussergewöhnlichen Untersuchungsinstrumentarien keinesfalls die Eröffnung einer weiteren intellektuellen Spielwiese etwa für Philosoph/innen oder andere Geisteswissenschaftler/innen intendiert ist. Das wäre ein grobes Missverständnis die Adressat/innen unserer methodologischen Erzeugnisse betreffend. Die konzipierten experimental- und imaginativhermeneutischen Verfahren und Techniken sind als methodische Angebote zu verstehen, die im Speziellen für therapieschulenforschende Absolvent/innen der SFU Wien gedacht sind, die darüber hinaus aber sehr wohl von allen an reflexionswissenschaftlicher Theorieforschung interessierten Psychotherapeut/innen für die Planung, Gestaltung und systematische Durchführung derartiger Analyseprojekte unbedingt genutzt werden sollten, damit die kritischreflexive Erkenntnisproduktion in einem noch grösseren Ausmass ins Rollen kommen kann.

\section{Wie Experimentalhermeneutische Laborforschung praktisch funktioniert}

Ein nun folgendes dialogexperimentelles Durchführungsbeispiel soll veranschaulichen, wie experimentalhermeneutisches Forschen zwischen psychotherapeutischen Disziplinen an der SFU Wien funktioniert. Im Zuge dieser sogenannten Dialogoperation werden die standardisierten Regelschritte des Verfahrens der Experimentellen TransKontextualisation (ExTK im TSD) auf die Konfrontation zwischen der «Strukturalen Psychoanalyse» (nach Jacques Lacan) und der «Transaktionsanalyse» (nach Eric Berne) angewandt. Dabei wird speziell das Diskursfeld Therapietheorie unter die Therapieschulen-interdisziplinäre Lupe genommen. Soll die wissenschaftshermeneutische Erkenntnisproduktion auf dem Gebiet der Psychotherapie tatsächlich auf überzeugende Art und Weise in Gang kommen, dann müssen selbstverständlich sämtliche therapieschulenkonstitutive Diskursfelder, wie etwa das anthropologische, das ethische, das sexualtheoretische, das psychopathologische Diskursfeld etc., in viele theorienreflexive Erkundungsdurchgänge involviert werden. Dieser wichtige Hinweis soll klar machen, dass die hier entwickelte Demonstration freilich nur einen ersten, vagen Eindruck von der Funktion der experimentalhermeneutischen PTW vermitteln kann. Darüber hinaus besteht der Zweck dieser Modellanalyse allein in der Illustration des strukturellen Prozesses einer wissenschaftshermeneutischen Untersuchung; nicht zuletzt deshalb, weil sie vom Methodenentwickler selbst und damit von einem Nicht-Therapeuten durchgeführt wurde.

Psychotherapeutisches Handeln im Sinne der Strukturalen Psychoanalyse ist hinsichtlich des Therapieziels an der «Freilegung eines hinter dem sich wissenden Ich verborgenen anderen Subjekts» interessiert. Diese zentrale Grundauffassung, die als ein charakteristisches Strukturelement der struktural-psychoanalytischen Disziplin betrachtet werden kann (Lacan; zit. n. Pagel, 1991, S. 24), wird zu exemplarischen Zwecken versuchsweise in die psychotherapeutische Disziplin der Transaktionsanalyse trans-kontextualisiert (verfremdungstechnisch übertragen). ${ }^{1}$ Damit ist folgende Festlegung getroffen:

> Herkunftskontext (HK): Als «eigene» Therapiedisziplin, die es hier kritisch-reflexiv zu erkunden gilt, wird im vorliegenden Beispiel die Strukturale Psychoanalyse betrachtet.

$>$ Verfremdungskontext (VK): Als heteromorphe Therapiedisziplin, in dessen Strukturgefüge eine Experimentelle Trans-Kontextualisation (ExTK) durchgeführt werden soll, dient die Transaktionsanalyse.

$>$ Diskursfeld (Df): Im Herkunftskontext wird ein Transponat (ein zu übertragendes Theorieelement) aus dem Grundlagenbereich «Therapietheorie» entnommen.

\section{A) Auswahl und Bestimmung des Transponats (T)}

$>$ Transponat (T): In der struktural-psychoanalytischen Therapie geht es um die «Freilegung eines hinter dem sich wissenden Ich verborgenen anderen Subjekts» (Lacan; zit. n. Pagel 1991, S. 24).

- Integrationsfreundlich-anmutender Transponats-Aspekt (ifa TA): In der struktural-psychoanalytischen Therapie geht es um die «Freilegung eines [...] verborgenen anderen Subjekts»/«sich wissendes Ich» (ebd.).

Hinweis: Aus bearbeitungstechnischen Gründen wird der ifa TA in diesem Beispiel in Aspektteil a) «sich wis-

1 Dieses hier leicht strukturmodifizierte Durchführungsbeispiel ist in seiner ursprünglichen Form abgedruckt in Greiner (2012, S. 242-253). 
sendes Ich» und Aspektteil b) «In der Therapie geht es um die Freilegung eines verborgenen anderen Subjekts» untergliedert. Folglich werden die Kurzexplikation der Herkunftsstruktur des ifa TA sowie dessen Übertragung in den Verfremdungskontext in zwei gesonderten Schritten versucht.

> Integrationsfraglicher Transponats-Aspekt (if TA): Das ist ein «hinter dem sich wissenden Ich verborgenes anderes Subjekt» (ebd.).

\section{B.a) Kurzexplikation des integrationsfreundlich- anmutenden Transponats-Aspekts (ifa TA/a) in seinem originalen Strukturzusammenhang}

Das «sich wissende Ich» bezeichnet Lacan in Anlehnung an Freuds Strukturbegriff des «Ich» auch als «moi» und bezieht sich mit diesem Terminus auf jenes egologische Phänomen, jene «reflexive» Form des «Ich», das «sich weiß» und das «sich denkt» (Pagel, 1991, S. 24). Im Zusammenhang mit der frühkindlichen Herausbildung und Ausgestaltung des «sich wissenden Ichs» spricht Lacan vom sogenannten «Spiegelstadium», das die «Matrix aller identifikatorischen Prozesse» repräsentiert. Bei diesem Entwicklungsvorgang des «Sich-Selbst-Hervorbringens des Ichs» soll der Begriff des Spiegels vor allem als «Modell für die Deskription einer imaginären Intersubjektivität» verstanden werden (ebd., S. 33f.).

\section{C.a) Übersetzung und Einbau}

\section{C.a.a) Auffinden und Vorstellen einer Heterokontextuellen Kopplung für den ifa TA/a}

Lacans Begriff des "sich wissenden Ichs", mit dem also jene Ich-Struktur angesprochen wird, die «sich weiß» und «sich denkt», soll nun in Verbindung gebracht werden mit dem transaktionsanalytischen Terminus des «integrierten Erwachsenen-Ich-Zustands» bei Berne. Springer (1994, S. 94) erklärt, dass sich das «integrierte Erwachsenen-Ich» bei miteinander zufriedenstellend kommunizierenden Menschen «in ihrem Fühlen für sich und andere, in ihrem klaren, uneingeschränkten Denken über sich, über Situationen und Menschen, in einem realitätsangemessenen Verhalten zu sich und anderen manifestiert.»

- Heterokontextuelle Kopplung für den ifa TA/a: Das «integrierte Erwachsenen-Ich» manifestiert sich bei Menschen «in ihrem klaren, uneingeschränkten Denken über sich, über Situationen und Menschen, in einem realitätsangemessenen Verhalten zu sich und anderen» (ebd.).

\section{C.a.b) Kurzexplikation der Heterokontextuellen Kopplung in ihrem originalen strukturzusammenhang}

Transaktionsanalytische Psychotherapeut/innen sprechen vom «Kindheits-, Eltern- sowie Erwachsenen-Ich» und beziehen sich mit dieser speziellen Ich-Zustands-Differenzierung auf drei unterscheidbare Kategorien menschlichen Denkens, Fühlens und Handelns. Die kindhaften Aktionsund Reaktionsformen des "Kindheits-Ich-Zustands", die elterlichen Denk- Fühl- und Handlungsweisen des «Eltern-Ich-Zustands» sowie die erfahrungsbezogenen und realitätsorientierten Verhaltens- und Aktivitätsmöglichkeiten des «Erwachsenen-Ich-Zustands» stellen als wesentliche Persönlichkeitsbestandteile nach Berne (1975, S. 24) bei jedem Menschen lebenslänglich wirksame Funktionsbereiche dar. Springer (1994, S. 94) fokussiert den letztgenannten Ich-Zustand und schreibt:

\begin{abstract}
«Wann immer Menschen zufriedenstellend miteinander auskommen, tun sie das, in der Sprache der Transaktionsanalyse ausgedrückt, aus einem integrierten Erwachsenen-Ich-Zustand heraus. In ihrem Fühlen für sich und andere, in ihrem klaren, uneingeschränkten Denken über sich, über Situationen und Menschen, in einem realitätsangemessenen Verhalten zu sich und anderen manifestiert sich dieses integrierte Erwachsenen-Ich.»
\end{abstract}

\section{C.a.c) Demonstration der Schnittmenge im Diskursfeld (Df)}

Ein funktionierender trans-kontextueller Brückenschlag zwischen den heterokontextuellen Begriffsformen der beiden Dialogpartner («sich wissendes Ich» bei Lacan und «integrierter Erwachsenen-Ich-Zustand» bei Berne) wird im Umstand sichtbar, dass die Erscheinungsweise des «klaren, uneingeschränkten Denkens über sich» (Springer), von dem transaktionsanalytische Therapeut/innen sprechen, für die egologische Figur des «sich wissenden Ichs» (reflexives «moi») im struktural-psychoanalytischen Kontext konstitutiv zu sein scheint. Jetzt bleibt eigentlich nur noch zu überprüfen, ob sich auch im transaktionsanalytischen Verständnis hinter diesem «sich wissenden Ich» (Lacan), das sich bei Berne im «integrierten ErwachsenenIch-Zustand» zeigt, «ein anderes Subjekt verbirgt», von dessen Existenz man in der struktural-psychoanalytischen Disziplin weiss und das es in der psychotherapeutischen Arbeit schliesslich «freizulegen» gilt.

\section{B.b) Kurzexplikation des integrationsfreundlich- anmutenden Transponats-Aspekts (ifa TA/b) in seinem originalen Strukturzusammenhang}

Bei Lacans «sich wissendem Ich», dem reflexiven «moi», handelt es sich um ein «fiktionales Ich». Strukturalpsychoanalytische Therapeut/innen machen nun dieses "sich wissende Ich", das durch eine "von Eigenliebe beherrschte Form der Intersubjektivität» charakterisiert ist, für jene «Distanz» verantwortlich, die das Subjekt von seinem «eigentlichen, irreflexiven Ich» trennt. Dieses «wahre Ich» wird von Lacan als «je» oder auch als «sujet de l'inconscient» bezeichnet, das als «Subjekt des Unbewussten» nie unmittelbar präsent ist (Pagel, 1991, S. 40f., 51). Psychotherapeut/innen des Lacani- 
anischen Lagers haben also zunächst sorgfältigst zwischen der sich entfaltenden Rede des imaginären «moi» ( «sich wissendes Ich») und einer verborgenen «anderen Rede» des Unbewussten zu unterscheiden, um jenes «es spricht» überhaupt vernehmen zu können, in dem sich das «wahre» Subjekt des «je» («verborgenes anderes Subjekt») artikuliert (ebd., S. 118). Schliesslich geht es in der struktural-psychoanalytischen Therapie darum, «das Subjekt zu seinem eigentlichen Sein bzw. zu seinem Seinsverfehlen in der Sprache zu führen [...] und die Wahrheit hinter dem falschen Objektivismus hervortreten zu lassen» (ebd., S. 122f.).

\section{C.b) Übersetzung und Einbau}

\section{C.b.a) Auffinden und Vorstellen einer Heterokontextuellen Kopplung für den ifa TA/b}

Der ifa $\mathrm{TA} / \mathrm{b}$ «In der Therapie geht es um die Freilegung eines verborgenen anderen Subjekts» wirft nun die Frage auf, ob im transaktionsanalytischen Denken ein Korrelat für Lacans «verborgenes anderes Subjekt» existiert, das sich vom "sich wissenden Ich» unterscheidet und das es für therapeutische Zwecke «freizulegen gilt». Das «Erwachsenen-Ich» im Sinne Bernes muss nicht in jedem Fall gut funktionieren und einen «integrierten Zustand» aufweisen. Wenn dieser spezielle Ich-Bezirk blockiert und nicht leistungsfähig genug ist, dann sprechen transaktionsanalytische Therapeut/innen von einer "Trübung des Erwachsenen-Ichs». Mögliche Ursachen für diese «Trübung» werden im strukturellen Kontext der beiden anderen Ich-Sektoren aufgestöbert, das heisst, in der Hypertrophie gewisser Kindheits-Ich- und/oder ElternIch-Aspekte erkannt. Die störende Wirkungsdominanz dieser beiden Ich-Aspekte gilt es mittels transaktionsanalytischer Methoden sichtbar zu machen, um eine therapeutische «Befreiung des Erwachsenen-Ichs» zu erreichen, die wiederum die Voraussetzung für die Herstellung eines «integrierten» Status darstellt (Harris, 1995, S. 119ff., 218f.). In diesen therapietheoretischen Auffassungszusammenhang scheint der ifa TA/b «In der Therapie geht es um die Freilegung eines verborgenen anderen Subjekts» aus dem Herkunftskontext der struktural-psychoanalytischen Disziplin tatsächlich integrierbar zu sein.

- Heterokontextuelle Kopplung für den ifa TA/b: Mithilfe der Transaktionsanalyse gilt es, dominante Kindheits-Ich- und/oder Eltern-Ich-Aspekte sichtbar zu machen, damit die therapeutische Umwandlung eines «getrübten Erwachsenen-Ichs» in einen «integrierten Zustand» gelingen kann (ebd.).

\section{c.b.b) Kurzexplikation der Heterokontextuellen Kopp- lung in ihrem originalen Strukturzusammenhang}

Psychotherapeut/innen, die sich in der transaktionsanalytischen Disziplin bewegen, wissen aus ihrer analytischtherapeutischen Erfahrung, dass das qualitative Phänomen eines «integrierten Erwachsenen-Ich-Zustands» in der zwischenmenschlichen Kommunikation eher selten zu beobachten ist. Harris berichtet, dass «das ErwachsenenIch eines Menschen [...] durch alte Aufzeichnungen aus der Vergangenheit geschädigt sein» kann, und er weist darauf hin, dass es bei manchen Menschen bereits «so geschwächt [ist], dass sie nicht mehr weiterkommen. Sie sind durch wiederholtes Versagen verkrüppelt oder durch Schuldgefühle erstarrt. Häufig kommt es zu körperlichen Symptomen.» (Harris, 1995, S. 215). Exemplarisch führt er eine diagnostische Formulierung an, mit der er «Erwachsenen-Ich-geschwächte» Analysanden konfrontieren könnte: «Sie haben eine Menge NICHT-O.K. in Ihrem Kindheits-Ich, und Ihr Erwachsenen-Ich ist dadurch erheblich getrübt. Darum lässt es Sie zuweilen unangemessen reagieren und gibt Ihrem drohenden Eltern-Ich eine Gelegenheit, auf Ihr Kindheits-Ich einzuschlagen [...]»» (ebd., S. 219)

In der transaktionsanalytischen Perspektive wird ein Hauptgrund für das gestörte Zusammenspiel der differenten Ich-Funktionen in der sogenannten «Trübung» gesehen. Transaktionsanalytische Psychotherapeut/innen unterscheiden hierbei zwei verschiedene Verursachungsmöglichkeiten («Eltern-Ich-bedingt», «KindheitsIch-bedingt») und drei differente Erscheinungsweisen (Vorurteile, Einbildungen und Halluzinationen) der «Erwachsenen-Ich-Trübung». Harris (ebd., S. 120f.) erläutert zunächst

«die Trübung des Erwachsenen-Ichs durch liegengebliebene, ungeprüfte Daten aus dem Eltern-Ich, die als wahr objektiviert worden sind. Hierbei handelt es sich um Vorurteile. Auf diese Weise werden Dogmen [...] bei Transaktionen auf der Basis von Vorurteilen objektiviert, bevor sie durch Informationen über die Realität (Erwachsenen-Ich) kontrolliert werden können. Vorurteile entwickeln sich in der frühen Kindheit, wenn die beschützenden Eltern die nähere Untersuchung bestimmter Gegenstände unterbinden. Aus Furcht vor elterlichem Tadel wagt das Kind nicht, diese Untersuchungen dennoch anzustellen. [...] das Eltern-Ich in diesen Menschen hat einen Teil ihres Erwachsenen-Ichs fest im Griff, und sie umgeben ihren voreingenommenen Standpunkt mit allen möglichen und unmöglichen Argumenten, nur um bei ihrer Einstellung bleiben zu können.»

Die zweite Verursachungsmöglichkeit der ErwachsenenIch-Trübung erfolgt

«durch das Kindheits-Ich mit Gefühlen oder archaischen Erlebnissen, die - vollkommen unangemessen - in der Gegenwart objektiviert werden. Die beiden häufigsten Symptome dieser Art der Trübung sind Einbildungen und Halluzinationen. Eine wahnhafte Einbildung beruht auf Furcht. [...] Eine Halluzination ist ein Phänomen, das durch äußersten Stress hervorgerufen wird und bei dem eine frühere äußere Erfahrung - Erniedrigung, Zurückweisung, Tadel - wieder äußerlich erlebt wird, obwohl keiner da ist»» (ebd., S. 121f.). 
Von Bernes «integriertem Erwachsenen-Ich-Zustand» als hypothetisches Korrelat für Lacans «sich wissendes Ich» unterscheiden sich natürlich die potenziell «Erwachsenen-Ich-einschränkenden» Ich-Zustände sowohl des «Eltern-Ichs» (Vorurteile), als auch des «Kindheits-Ichs» (Einbildungen und Halluzinationen). Beide Ich-Aspekte (Eltern-Ich und Kindheits-Ich) sind als mögliche Störungsquellen zwar «intra-psychisch» bzw. «intra-personell» wirksam, ohne aber deshalb vom betroffenen Subjekt ausserhalb der analytischen Situation jedenfalls - reflexiv wahrgenommen, das heisst bewusst registriert werden zu können. Für weitere therapeutische Interventionen sind sie zuerst einmal über den transaktionsanalytischen Zugang «freizulegen» und «sichtbar zu machen».

\section{C.b.c) Demonstration der Schnittmenge im Diskursfeld (Df)}

In der Tat besteht für Lacans Konstruktion eines «irreflexiven Ichs», eines vom "sich wissenden Ich» unterscheidbaren «anderen Subjekts» eine potenzielle Integrationschance in Bernes Therapieterrain. Speziell der Strukturaspekt spezifischer Persönlichkeits-Segmente (Eltern-Ich-Zustand, Kindheits-Ich-Zustand), deren ontogenetisch bedingte qualitative Beschaffenheit als unreflektierbare Ursache für die Verhinderung einer realitätsangepassten Ich-FunktionsEntwicklung («integrierter Erwachsenen-Ich-Zustand») identifiziert werden kann, bietet im transaktionsanalytischen Feld eine trans-kontextuelle Übersetzungsmöglichkeit für Lacans Figur eines «verborgenen anderen Subjekts». Die (dominante) Existenz eines solchen «verborgenen anderen Subjekts» lässt sich in Bernes Kontext demnach in der hypertrophen Ausprägung der Eltern-Ich- und/oder Kindheits-Ich-Instanz transaktionsanalytisch aufweisen vorausgesetzt allerdings, das Erwachsenen-Ich zeigt sich als geschwächt, geschädigt, nicht-integriert. Der ifa TA/b «In der Therapie geht es um die Freilegung eines verborgenen anderen Subjekts» aus der struktural-psychoanalytischen Disziplin soll nun im transaktionsanalytischen Revier folgendermassen angewandt werden: In vielen Fällen «verbirgt» sich ein potenziell zweigliedriges «anderes Subjekt» (dominanter Kindheits-Ich- und/oder Eltern-Ich-Aspekt), das es zu therapeutischen Zwecken unbedingt dann «freizulegen» gilt, wenn es das «sich wissende Ich» (integrierter Erwachsenen-Ich-Zustand) in seiner vollen Funktionsweise blockiert bzw. «trübt» (durch Eltern-Ich-bedingte «Vorurteile» und/oder Kindheits-Ich-bedingte "Einbildungen», «Halluzinationen») und damit einen «nicht-integrierten Erwachsenen-Ich-Zustand» verursacht.

\section{D) Kritische Testung des heterokontextuellen Integrationsversuchs}

\section{D.a) Fokus auf den integrationsfraglichen Aspekt des Transponats (if TA)}

Im ausgewählten Transponat aus dem Herkunftskontext der Strukturalen Psychoanalyse «verbirgt» sich die- ses «andere Subjekt» hinter dem «sich wissenden Ich» (if TA). Mit der Thematisierung des integrationsfraglichen Transponats-Aspekts (ein hinter dem «sich wissenden Ich» verborgenes anderes Subjekt) wird jetzt folgende Frage spannend: Welche Position nimmt in der transaktionsanalytischen Denktradition das potenziell zweigliedrige «andere Subjekt» (dominanter Kindheits-Ich- und/oder Eltern-Ich-Aspekt) im Verhältnis zum «sich wissenden Ich» (integrierter Erwachsenen-Ich-Zustand) ein? Lassen sich - analog zu Lacans theoretischer Perspektive - «dominante Kindheits-Ich- und/oder Eltern-Ich-Aspekte» (anderes Subjekt) hinter dem «integrierten ErwachsenenIch-Zustand» (sich wissendes Ich) auffinden?

\section{D.b) Überprüfung der heterokontextuellen Übertragungseignung angesichts des if TA}

Zur Rekonstruktion: Unter C.b.c) wird zunächst behauptet, dass sich die (dominante) Existenz eines solchen «verborgenen anderen Subjekts» in Bernes Kontext in der hypertrophen Ausprägung der Eltern-Ich- und/oder Kindheits-Ich-Instanz transaktionsanalytisch aufweisen lässt. Es wird aber gleichzeitig auch auf die notwendige Bedingung für diese spezifische Konstellation hingewiesen. Nur unter der Voraussetzung, dass sich der Erwachsenen-Ich-Zustand als geschwächt, geschädigt, das heisst nicht-integriert darstellt, macht die Bemühung um eine «Freilegung eines verborgenen anderen Subjekts» im Feld der Transaktionsanalyse überhaupt Sinn. Exakt an dieser Stelle zeigt sich jetzt eine unüberbrückbare Kluft zwischen Lacans und Bernes Ansatz. Funktioniert nämlich das "sich wissende Ich» (Lacan) in der Form eines «integrierten Erwachsenen-Ich-Zustands» (Berne) zufriedenstellend, dann erweist sich die Intention, ein «verborgenes anderes Subjekt freizulegen", als Absurdität. In diesem Ich-Funktions-Status nach einem «anderen Subjekt» zu suchen, wirkt deshalb widersinnig, weil sich Kindheits-Ich- und/oder Eltern-Ich-Aspekte infolge der Erwachsenen-Ich-Konsolidierung auch nicht als «anderes Subjekt» artikulieren. Transaktionsanalytisch betrachtet kann demnach gar kein «anderes Subjekt» hinter einem «integrierten Erwachsenen-Ich-Zustand» («sich wissendes Ich») existieren, das es freizulegen gälte. Im Falle einer «Erwachsenen-Ich-Trübung» sind Kindheits-Ich- und/ oder Eltern-Ich-Aspekte («anderes Subjekt») dermassen «dominant», dass sie vielmehr anstelle eines «integrierten Erwachsenen-Ich-Zustands» («sich wissendes Ich») treten. > Präsentation des Extrabierten Kontradikts (Ort des Widerspruchs): In der transaktionsanalytischen Disziplin spricht man vom Phänomen der «Trübung des Erwachsenen-Ichs», das mit dem häufig anzutreffenden Umstand zusammenhängt, dass «das Erwachsenen-Ich eines Menschen [...] durch alte Aufzeichnungen aus der Vergangenheit geschädigt sein» kann. Tatsächlich ist es «bei manchen Menschen [...] so geschwächt, dass sie nicht mehr weiterkommen. Sie sind durch wiederholtes Versagen verkrüppelt oder durch Schuldgefühle erstarrt. Häufig kommt es zu körperlichen Symptomen» (Harris, 1995, S. 215). 


\section{D.c) Heterokontextueller Übertragungsbruch am Kontradikt}

Auch im psychotherapeutischen Kontext nach Berne ist man darum bemüht, ein vom «sich wissenden Ich» («integrierter Erwachsenen-Ich-Zustand») unterscheidbares «anderes Subjekt» («dominante Kindheits-Ich- und/oder Eltern-Ich-Aspekte») in therapeutischer Absicht über analytische Zugänge in den Griff zu bekommen. Allerdings wird diese Unternehmung nur dann notwendig und auch möglich, wenn sich das Erwachsenen-Ich der/s Klientin/ en infolge einer «Trübung» als «nicht integriert» erweist. Erst wenn der «integrierte Status» des Erwachsenen-Ichs «trübungsbedingt» aufgelöst ist, lässt sich ein «anderes Subjekt» transaktionsanalytisch nachweisen, weil «dominante Kindheits-Ich- und/oder Eltern-Ich-Aspekte» immer nur anstelle des "sich wissenden Ichs» existieren. An diesem Sachverhalt bricht der transkontextuelle Integrationsversuch definitiv. Die struktural-psychoanalytische Transponatsaussage aus dem Diskursfeld «Therapietheorie» nach Lacan «In der Therapie geht es um die Freilegung eines hinter dem «sich wissenden Ich〉 verborgenen anderen Subjekts» $(\mathrm{T})$ müsste demnach modifiziert werden in die Formulierung «In der Therapie geht es um die Freilegung eines anstelle des «sich wissenden Ichs〉 verborgenen anderen Subjekts», damit sie in die transaktionsanalytische Disziplin integrierbar wird. Pointiert lässt sich behaupten, dass die struktural-psychoanalytischen Interventionen überhaupt erst dann beginnen können, nachdem der/die Psychotherapeut/in im Sinne Bernes seine/ihre transaktionsanalytisch-therapeutische Arbeit erfolgreich abgeschlossen hat. Erst wenn ein «integrierter Erwachsenen-Ich-Status» erreicht ist, kann man hinter diesem «sich wissenden Ich» mit der «Freilegung» eines «verborgenen anderen Subjekts» im Sinne Lacans analytisch ansetzen.

Wie auch immer, Faktum ist, dass sich in diesem Dialogexperiment tatsächlich ein unüberwindlicher Spalt, eine fundamentale Heterogenität im therapietheoretischen Anspruch der beiden psychotherapeutischen Ansätze offenbart, weshalb man auch ein Scheitern der struktural-psychoanalytischen Zielsetzung im Kontext der methodologischen Rahmenbedingungen nach Berne konstatieren muss.

\section{E) Reflexionsprofit}

Struktural-psychoanalytische Therapeut/innen können aber aus der diskursiven Begegnung mit der transaktionsanalytischen Denktradition lernen, dass selbst das «sich wissende Ich», das «reflexive moi» - so «imaginär» und «fiktional» es auch immer sein mag - eine strukturelle Plastizität, eine qualitative Form- und Veränderbarkeit aufweist, die man mit analytischen Zugängen und therapeutischen Mitteln beeinflussen kann und auch muss, weil der Status quo des «bewussten Ichs», das «sich denkt» und «sich weiß», für das Zustandekommen und für den gelingenden Prozess zwischenmenschlichen Kommunizierens, für die Produktion von erfolgreich funktionierender Intersubjektivität in der Lebenswelt höchstrelevant ist. Potenziell könnte eine Erfahrung bzw. ein gedanklicher Impuls dieser Art zu theoriekritischen Auseinandersetzungen mit den konzeptuellen Bedingungen des eigenen Therapieverständnisses anregen, die sich möglicherweise sogar auf die Genese therapiemethodologischer Innovationsschritte auswirken.

\section{F) Zusammenfassung der Dialogresultate}

Ergebnisse der dialogischen Konfrontation zwischen Therapiedisziplin Strukturale Psychoanalyse (HK) und Therapiedisziplin Transaktionsanalyse (VK) im Diskursfeld (Df) Therapietheorie am Transponat (T) «In der Therapie geht es um die Freilegung eines hinter dem sich wissenden Ich verborgenen anderen Subjekts.» (Lacan in Pagel, 1991, S. 24) gemäss der verwendeten Datenbasis.

\section{F.a) Transponats-relative Schnittmenge im Diskursfeld (Df) Therapietheorie gemäss der verwendeten Datenbasis}

In Bezug auf das Transponat lässt sich folgende Gemeinsamkeit zwischen der struktural-psychoanalytischen (Lacan) und der transaktionsanalytischen (Berne) Disziplin feststellen: Sowohl im therapeutischen Kontext Lacans als auch Bernes rechnet man mit der psychischen Wirklichkeit einer reflexiven Ich-Struktur, die «sich weiß» und «sich denkt». Ebenso kennt man in beiden psychotherapeutischen Disziplinen eine davon unterschiedene andersartige egologische Gestalt, deren Wirkungszusammenhänge ohne analytische Interventionen nicht reflexiv einholbar sind, das heisst, deren Strukturmerkmale gewissermassen im «Verborgenen» liegen und in der analytisch-therapeutischen Arbeit zuerst einmal freigelegt bzw. auf die Ebene einer bewussten Auseinandersetzung gehoben werden müssen.

\section{F.b) Transponats-relative Differenz im Diskursfeld (Df) Therapietheorie gemäss der verwendeten Datenbasis}

Der transponats-bezogene Unterschied zwischen den beiden psychotherapeutischen Disziplinen (Lacan und Berne) muss nun in der Position verortet werden, die die beiden Ich-Figuren im Verhältnis zueinander jeweils einnehmen. Während struktural-psychoanalytische Therapeut/innen darum bemüht sind, die verborgene Ich-Figur «hinter» der reflexiven Form des Ichs freizulegen, sind transaktionsanalytische Therapeut/innen damit beschäftigt, eine verborgene (potenziell zweigliedrige) Ich-Struktur aufzudecken, die aufgrund ihrer dominanten Ausprägung adäquat funktionierende reflexive Aktivitäten verunmöglicht und damit anstelle dieser selbstbezüglichen Gestalt des Ichs tritt.

\section{F.c) Transponats-relativer Reflexionsprofit für die Strukturale Psychoanalyse (HK)}

Der spezifische, transponats-bezogene Erkenntnisgewinn lässt sich im gescheiterten trans-kontextuellen Integrati- 
onsversuch für struktural-psychoanalytische Therapeut/ innen in folgendem potenziellen Erfahrungszuwachs ausmachen: Es gibt jede Menge grundlegende therapeutische Arbeit zu leisten, noch bevor man mit analytischen Mitteln die individualhistorisch nuancierte, in sozialisationsgeschichtliche Bedingungen eingebettete, auf soziokulturell vorgeprägten Mustern basierende Struktur der Selbstverständlichkeit einer/s Klientin/en in strukturalistischer Manier aufzulösen beginnt. Die Erscheinungsweisen des selbstverständlichen Sich-und-die-Welt-Kennens sind vielfältige, mehr oder weniger gut funktionierende, zumeist aber beschädigte, sodass es selbst im reflexiven Diesseits noch viel genuin Psychotherapeutisches zu tun gibt. Schliesslich setzt eine zufriedenstellende Form des zwischenmenschlichen Sich-Begegnens in der psychosozialen Lebenswelt notwendigerweise ein funktionstüchtiges «sich wissendes Ich» voraus.

\section{Auf dem psychotherapiewissenschaftlichen Weg zur Akademischen Psychotherapie}

Es zeigt sich heute, dass sich nicht nur das älteste und damit früherprobte Therapieschulen-interdisziplinäre bzw. inter-therapeutische Analyseverfahren der Experimentellen Trans-Kontextualisation (ExTK im TSD), dessen Funktionsweise anhand der soeben durchgeführten Modellanalyse vorgestellt wurde, in vielen SFUForschungsprojekten erfolgreich bewähren konnte (Bartl, 2016; Greiner et al., 2009, 2010; Greiner, 2011, 2012, 2013a; Greiner \& Jandl, 2012; Seggl, 2018; Tichy, 2018), sondern dass sich die wissenschaftspraktische Tauglichkeit der jüngeren, bizarrosophischen Methoden und Techniken ebenso belegen lässt. Immerhin liegen hierzu mittlerweile neben einigen paradigmatischen Durchführungsbeispielen auch mehrere vorzeigbare approbierte SFU-Qualifizierungsstudien vor (Greiner \& Jandl, 2012, 2015; Greiner et al., 2013; Greiner, 2013a-d, 2014). Insgesamt kann aktuell auf über 50 Graduierungsschriften verwiesen werden, die sich auf alle drei akademische Niveaus verteilen (Bakkalaureat, Magisterium, Doktorat), in denen mit experimental- und imaginativhermeneutischen Instrumentarien produktiv geforscht wurde. Da im Verlauf der vergangenen zwölf Jahre die Nützlichkeit unserer irritationslogischen Reflexionstechniken und radikalkreativen Untersuchungsmethoden für die therapieschulenanalytische Grundlagenforschungspraxis offenkundig geworden ist, dürfen wir auch behaupten, dass wir mit unserem speziellen SFU-Programm der Experimental- und Imaginativhermeneutischen PTW einen konstruktiven Beitrag zum Akademisierungsprozess, zur Statuskarriere der Psychotherapie zu leisten imstande sind. Wir werden in die eingeschlagene Richtung jedenfalls weitermarschieren, weil vieles dafür spricht, dass wir uns damit auf dem besten Weg zur Akademischen Psychotherapie befinden.

Mit unserem Ansatz einer wissenschaftshermeneutisch konzeptualisierten PTW garantieren wir die methodischen Möglichkeiten, um in die komplexen Voraussetzungsstrukturen psychotherapeutischer Aktivitäten vertiefte
Einsichten zu gewinnen, und stellen so die kritisch-reflexive Erkenntnisproduktion für das einzelwissenschaftliche Fach Psychotherapie sicher. Verzichtet Psychotherapie aber auf die praktische Umsetzung dieses 2. Konstitutionskriteriums für akademische Wissenschaftlichkeit und begnügt sich damit, funktionales Anwendungswissen für die Therapiepraxis zu schaffen, verkürzt sie sich selbst auf die Ebene des technischen Handelns. Entwicklung und Ausbildung des instrumentellen Funktionsniveaus sind natürlich von essenzieller Bedeutung. Auch Erismann (2019, S. 16 i.d. Heft) bezieht sich auf das therapeutische Techniklevel, wenn er behauptet, dass in der Psychotherapie (wobei er selbst von PTW spricht und damit das meint, was wir unter PT verstehen) «der gemeinsame, dialogische Reflexionsprozess von PatientIn und TherapeutIn Ort des therapeutischen Heilens und der Wissensgenerierung» ist. Wer jedoch die Wissenschaftlichkeit der Psychotherapie allein an diesem 1. Konstitutionskriterium (technisch-funktionales Wissen) festzumachen versucht, der muss sich darüber auch im Klaren sein, dass damit noch kein überzeugendes wissenschaftstheoretisches Abgrenzungsmerkmal gegenüber anderen, mehr oder weniger methodenbasierten Wissens- und Praxisformen genannt ist. Wie auch immer die «Methode der Reflexion» (Erismann) theoretisch konzeptualisiert und technisch ausdifferenziert sein mag - die VerstehensWerkzeuge des Auslegens, Deutens, Interpretierens und Reflektierens spielen nicht nur in der Psychotherapie eine zentrale Rolle, sondern haben ebenso in sämtlichen esoterischen, spiritistischen, fremdkulturellen etc. Praxen wissensgenerierende Funktion (1. Konstitutionskriterium), ohne dass diese Praxen dabei als Akademische Wissenschaften (1.+2. Konstitutionskriterium) gelten.

\section{Literatur}

Bartl, A. (2016). «Der regressive Prozess» im therapieschulenübergreifenden Dialog. Eine Experimentelle Trans-Kontextualisation (ExTK) zwischen Psychoanalyse und Gestaltherapie. Psychoanalyse und Körper, 15(I/II), 61-84/81-110.

Berne, E. (1975). Was sagen Sie, nachdem Sie «Guten Tag» gesagt haben? Psychologie des menschlichen Verhaltens. München: Kindler TB.

Erismann, M. (2019; i.d. Heft). Der Wissenschaftsbegriff der Psychotherapiewissenschaft. Psychotherapie-Wissenschaft, 9(2), 13-17.

Greiner, K. (2007). Psychoanalytik als Wissenschaft des 21. Jahrhunderts. Ein konstruktivistischer Blick auf Struktur und Reflexionspotential einer polymorphen Kontextualisations-Technik. Frankfurt/M: Peter Lang.

Greiner, K. (2011). Integrationsprogramm Therapieschulendialog (TSD). Entwicklung einer textanalytischen Grundlagenforschung in der Psychotherapiewissenschaft. Frankfurt/M: Peter Lang.

Greiner, K. (2012). Standardisierter Therapieschulendialog (TSD). Therapieschulen-interdisziplinäre Grundlagenforschung an der Sigmund-Freud-Privatuniversität Wien/Paris (SFU). Wien: SFU-Verlag.

Greiner, K. (2013a). Methodenfahrplan Inter-Therapeutik (ITK). Transfermeneutische Psychotherapiewissenschaft an der SFU Wien. Wien: SFU-Verlag.

Greiner, K. (2013b). Psycho-Text-Puzzle: Theoriendiskursives Spielverfahren für die Psychotherapiewissenschaft. In F. Lan, F. G. Wallner \& A. Schulz (Hrsg.), Concepts of a Culturally Guided Philosophy of Science. Contributions from Philosophy, Medicine and Science of Psychotherapy (S. 85-107). Frankfurt/M: Peter Lang. 
Greiner, K. (2013c). Psycho-Bild-Prozess (PBP): Imaginationsförderndes Reflexionsinstrumentarium für die Psychotherapiewissenschaft. In F. Lan, F. G. Wallner \& A. Schulz (Hrsg.), Concepts of a Culturally Guided Philosophy of Science. Contributions from Philosophy, Medicine and Science of Psychotherapy (S. 109-126). Frankfurt/M: Peter Lang.

Greiner, K. (2013d). Intertherapeutischer Bild-Prozess (ITBP). Eine transfermeneutische Forschungstechnik der Psychotherapiewissenschaft. SFU Forschungsbulletin - SFU Research Bulletin, 1, 41-55.

Greiner, K. (2014). Interdisziplinäres Psycho-Text-Puzzle (P-T-P) am Beispiel Psychoanalyse kombiniert mit Wissenschaftstheorie. Eine experimentalhermeneutische Modellreflexion. SFU Forschungsbulletin - SFU Research Bulletin, 2, 1-15.

Greiner, K. (2015a). Psychotherapia Academica Universitatis: A Philosophical Argument for the Academic Discipline of Psychotherapy. In O. C. G. Gelo, A. Pritz \& B. Rieken (Hrsg.), Psychotherapy Research. Foundations, Process, and Outcome (S. 93-116). Wien: Springer.

Greiner, K. (2015b). Akademische Psychotherapie. Philosophische Fundierung einer neuen Einzelwissenschaft an der Sigmund-FreudPrivatuniversität Wien (SFU). In K. Greiner \& M.J. Jandl (Hrsg.), Bizarrosophie. Radikalkreatives Forschen im Dienste der akademischen Psychotherapie (S. 11-34). Nordhausen: Traugott Bautz.

Greiner, K. \& Jandl, M.J. (2010). Novum Therapieschulendialog (TSD): Methodologische Prinzipien einer theorien-integrativen Psychotherapiewissenschaft. In K. Greiner, M. J. Jandl \& F. G. Wallner (Hrsg.), Aus dem Umfeld des Konstruktiven Realismus. Studien zu Psychotherapiewissenschaft, Neurokritik und Philosophie. Culture and Knowledge, Bd. 14 (S. 15-41). Frankfurt/M: Peter Lang.

Greiner, K. \& Jandl, M.J. (2012). Das Psycho-Text-Puzzle und andere Beiträge zu Psychotherapiewissenschaft und Philosophie. Wien: SFU-Verlag.

Greiner, K. \& Jandl, M.J. (Hrsg.). (2015). Bizarrosophie. Radikalkreatives Forschen im Dienste der akademischen Psychotherapie. Nordhausen: Traugott Bautz.

Greiner, K. \& Jandl, M. J. \& Burda, G. (2013). Der Psycho-Bild-Prozess und andere Beiträge zu Psychotherapiewissenschaft und Philosophie. Wien: SFU-Verlag.

Greiner, K., Jandl, M.J. \& Paschinger, O. (Hrsg.). (2009). Programmatik und Praxis im Therapieschulendialog (TSD). Erste Beiträge zur dialogexperimentellen Theorien-Integration in der Psychotherapiewissenschaft. Wien: SFU-Verlag.

Greiner, K., Jandl, M.J. \& Wallner, F. G. (Hrsg.). (2010). Aus dem Umfeld des Konstruktiven Realismus. Studien zu Psychotherapiewissenschaft, Neurokritik und Philosophie. Culture and Know ledge, Bd. 14. Frankfurt/M: Peter Lang.

Harris, T. A. (1995). Ich bin O.K. Du bist O.K. Wie wir uns selbst besser verstehen und unsere Einstellung zu anderen verändern können. Eine Einführung in die Transaktionsanalyse. Reinbek/H: Rowohlt.

Jandl, M. J. (2006). Verfremdung als literarische Strategie: Beckett und Kafka interpretiert von Anders und Adorno. In K. Greiner, F. G. Wallner \& M. Gostentschnig (Hrsg.), Verfremdung - Strangification. Multidisziplinäre Beispiele der Anwendung und Fruchtbarkeit einer epistemologischen Methode (S. 113-142) Frankfurt/M: Peter Lang.

Pagel, G. (1991). Lacan zur Einführung. Hamburg: Junius.

Seggl, F. (2018). Gestalt-Therapie und Systemische Therapie im Dialog. Eine psychotherapiewissenschaftliche Untersuchung auf experimentalhermeneutischer Basis. Nordhausen: Traugott Bautz.

Springer, G. (1994). Transaktionsanalyse. In G. Stumm \& B. Wirth (Hrsg.), Psychotherapie - Schulen und Methoden. Eine Orientierungshilfe für Theorie und Praxis (S. 90-100). Wien: Falter.

Tichy, H.E. (2018). Die Kunst präsent zu sein. Carl Rogers und das frühbuddhistische Verständnis von Meditation. Münster: Waxmann.

Wagner, E. (1996). Psychotherapie als Wissenschaft in Abgrenzung von der Medizin. In A. Pritz A (Hrsg.), Psychotherapie-eine neue Wissenschaft vom Menschen (S. 219-247). Wien: Springer.

Wallner, F. G. (1992a). Acht Vorlesungen über den Konstruktiven Realismus. Wien: WUV.

Wallner, F. G. (1992b). Wissenschaft in Reflexion. Wien: Braumüller.

Wallner, F. G. (2002). Die Verwandlung der Wissenschaft. Vorlesungen zur Jahrtausendwende. Hamburg: Kovac.
Academic psychotherapy, psychotherapy science, and experimental hermeneutic lab research. A supplement to Markus Erismann's «Der Wissenschaftsbegriff der Psychotherapiewissenschaft" [The scientific notion of psychotherapy sciences]

This commentary on Markus Erismann's essay titled «Der Wissenschaftsbegriff der Psychotherapiewissenschaft» is designed as a supplement to the argumentation which intends to show what psychotherapy science designed in a scientific-hermeneutic manner could potentially contribute to the academic status of psychotherapy. Building on the theoretical discussion and methodological explanations, we demonstrate using a sample model analysis how this innovative fundamental research practice works, which can help advance psychotherapy into a genuine individual science.

Key words: academic psychotherapy, Markus Erismann, experimental and imaginative hermeneutic psychotherapy sciences, Greiner-Jandl initiative, therapeutic school research, therapeutic school dialog (TSD), experimental trans-contextualization (ExTK), Sigmund Freud PrivatUniversität Wien (SFU)

\section{Psicoterapia accademica, psicoterapia scientifica e ricerca di laboratorio sperimentale ermeneutica. Un'integrazione a «Der Wissenschaftsbegriff der Psychotherapiewissenschaft» di Markus Erismann}

Questo commento al saggio di Markus Erismann dal titolo «Der Wissenschaftsbegriff der Psychotherapiewissenschft» (Il concetto scientifico di scienza della psicoterapia) è concepito come un'integrazione argomentativa in cui deve essere mostrato ciò che una psicoterapia scientifica concettualizzata scientificamente-ermeneuticamente può potenzialmente fare per la carriera accademica della psicoterapia. A seguito di discussioni teoriche e spiegazioni metodologiche sulla base di un'analisi esemplare del modello, viene così dimostrato come funziona quella pratica innovativa di ricerca di base che può contribuire all'avanzamento della psicoterapia a vera scienza singola.

Parole chiave: psicoterapia accademica, Markus Erismann, psicoterapia scientifica ermeneutica sperimentale e immaginativa, programma Greiner-Jandl, ricerca delle scuole di terapia, Therapieschulendialog (TSD), Transconstestualizzazione sperimentale (ExTK), Sigmund Freud PrivatUniversität Wien (SFU)

\section{Der Autor}

Kurt Greiner ist Professor der Psychotherapiewissenschaft an der Sigmund Freud PrivatUniversität (SFU) in Wien, wo er seit 2007 im Fachbereich Psychotherapiewissenschaftstheorie und Therapieschulenforschungsmethodologie lehrt und forscht.

\section{Kontakt}

Univ.-Prof. DDr. Kurt Greiner

Sigmund Freud PrivatUniversität Wien (SFU)

Galerie-Office 4003, Campus Prater

Freudplatz 1,

A-1020 Wien

E-mail: kurt.greiner@sfu.ac.at 